
cultures

Les cahiers de l'Acedle

18-2 | 2021

Didactique des langues \& plurilinguisme(s) : 30 ans de recherches

\title{
Plurilinguisme(s) et appropriation du français par des adultes migrants
}

\section{Emilie Lebreton et Éric Mercier}

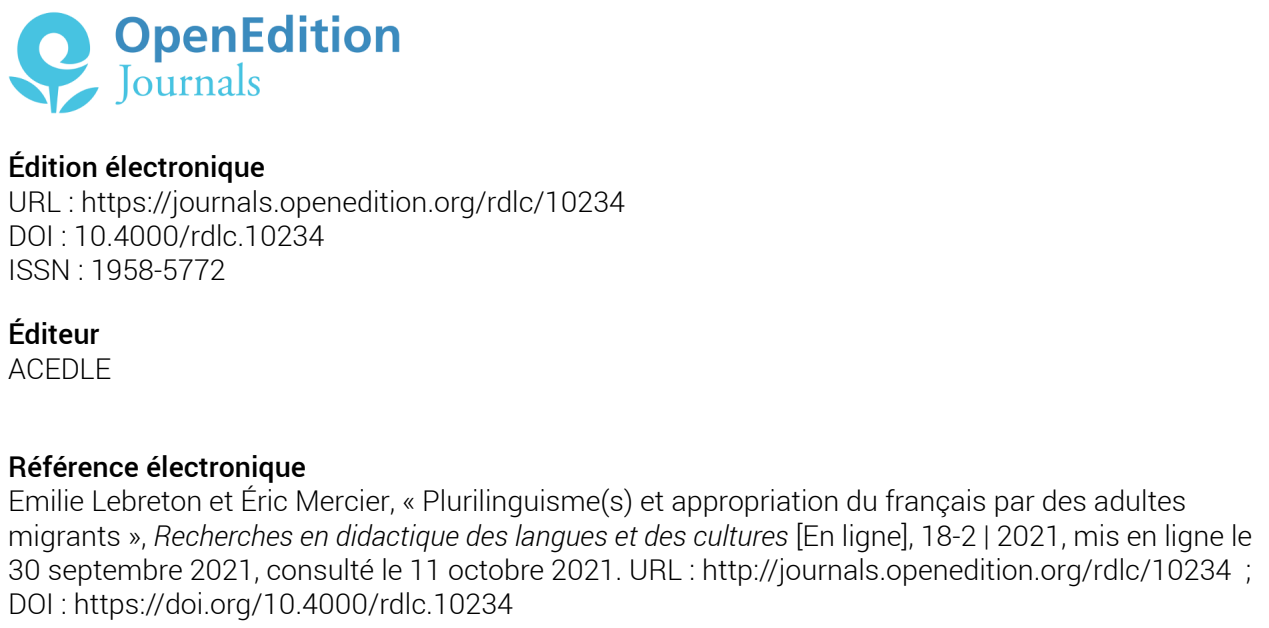

Ce document a été généré automatiquement le 11 octobre 2021.

\section{(c) (1) ()}

Recherches en didactique des langues et des cultures is licensed under a Creative Commons AttributionNonCommercial-NoDerivatives 4.0 International License 


\title{
Plurilinguisme(s) et appropriation du français par des adultes migrants
}

\author{
Emilie Lebreton et Éric Mercier
}

\section{Introduction}

1 En France, les formations linguistiques pour adultes migrants représentent un contexte formatif où le plurilinguisme n'est pas au centre des priorités des politiques formatives régissant ce champ, puisque l'exclusivité du français tend à s'imposer à la fois comme une évidence et une nécessité (Hambye et Romainville, 2014 ; Lebreton, 2017 ; OFII, 2019; Mercier, 2020). Il s'avère donc relativement difficile de mener une réflexion portant sur le plurilinguisme et sur la didactique du plurilinguisme, sans se confronter à la règle du monolinguisme qui est automatiquement rapportée à la perspective d'insertion de ces personnes. L'objectif de cet article est de s'interroger sur la place et le rôle accordés au plurilinguisme et au monolinguisme, et ce, au regard des réceptions d'apprenants et de pratiques formatives mises en œuvre. Il s'agit de comprendre comment les apprenants engagés dans ces dispositifs s'accommodent et appréhendent à la fois les discours circulants et les orientations didactiques favorisant une relative ouverture au plurilinguisme. En outre, c'est à l'appui de leurs réceptions et expériences que nous pourrons augmenter la réflexion sur une didactique du plurilinguisme pensée dans une perspective appropriative (au sens de Castellotti, 2017). Au-delà de la (seule) gestion des répertoires linguistiques et de l'outillage des locuteurs, l'appropriation d'une nouvelle langue relève d'une transformation existentielle où de nouveaux éléments s'insèrent dans l'histoire de la personne et modifient notre compréhension du monde. 


\section{Plurilinguisme et formations linguistiques des adultes migrants}

\section{Un climat d'ouverture à la diversité linguistique et au plurilinguisme}

2 Phénomène majeur de nos sociétés, le plurilinguisme est admis et soutenu par des décideurs politiques, des chercheurs et des professionnels issus du champ éducatif et formatif. Aujourd'hui, il n'est plus à démontrer que des institutions européennes, appuyées par des études scientifiques, affirment que le plurilinguisme constitue un indéniable atout économique, social et culturel tant pour l'individu que pour la société (Lüdi, 2002). La dynamique initiée par les travaux de Dabène et Coste, dès les années 1990 a contribué à mettre en lumière la pertinence et l'intérêt du plurilinguisme dans les situations d'enseignement-apprentissage, notamment en soulignant que chaque acteur social est traversé par la pluralité des langues et des cultures (Coste, 2010) et qu'il est ainsi précieux de privilégier une ouverture à l'altérité et à la diversité linguistique et culturelle dans le système éducatif. Depuis, le plurilinguisme tend à occuper le devant de la scène dans les recherches en didactique des langues. Les réflexions développées au cours de ces dernières décennies ont porté leurs fruits et se sont révélées bénéfiques à bien des égards: qu'il s'agisse de politiques linguistiques éducatives, du développement des compétences linguistiques et culturelles des apprenants ou de la sensibilisation et de la formation des enseignants aux enjeux de la diversité et du plurilinguisme. Les expérimentations et les travaux ont certes été essentiellement menés sur le terrain scolaire, mais les questions et les résultats qui en découlent alimentent notre réflexion sur ce qui se passe au sein des formations linguistiques pour adultes migrants. Il s'avère que pour ces personnes, et pour les dispositifs dans lesquels ils sont inscrits, la question du plurilinguisme se pose différemment. Ces adultes sont, de fait, considérés comme plurilingues. En effet, au gré de leurs parcours migratoires, ces personnes se sont constituées des répertoires langagiers composés de plusieurs langues ou de variétés linguistiques, dont les degrés de maitrise et les compétences diffèrent d'une langue à l'autre. La diversité linguistique et culturelle apparait alors comme constitutive des formations linguistiques destinées aux adultes migrants. À première vue, la simple présence de ces adultes migrants suffirait à induire que le plurilinguisme habite les formations linguistiques, et « qu'elles relèvent de l'éducation plurilingue » (Beacco, 2008:5). Si nous rejoignons l'idée d'une diversité et d'un plurilinguisme visibles, nous avons davantage de réserves et d'interrogations concernant le rapport établi avec l'éducation plurilingue. Toutefois, nous retenons ici une certaine ouverture à la diversité linguistique et au plurilinguisme pour un contexte éducatif qui n'apparait pas nécessairement concerné de prime abord par ces questions. Cette ouverture s'illustre par le projet sur l'intégration linguistique des migrants adultes (ILMA), lequel est porté depuis une dizaine d'années par des experts du Conseil de l'Europe (COE). C'est un exemple notable qui s'attache à souligner l'importance des langues d'origine et leur rôle dans les processus d'insertion des personnes (Beacco, 2008). Au-delà de la reconnaissance du plurilinguisme et de la mobilisation des compétences déjà-là, des outils et des activités ancrés dans une approche plurilingue et pluriculturelle sont rendus accessibles. En témoigne «le portrait plurilingue $»^{1}$ proposé dans la boite à outils du COE destinée à l'accompagnement linguistique des adultes réfugiés, qui est une activité de 
conscientisation des ressources linguistique emblématique de la didactique du plurilinguisme.

3 En outre, il est également admis par les auteurs d'un autre texte cadre faisant souvent autorité dans les formations linguistiques, le référentiel Français Langue d'Intégration, que l'apprentissage du français ne contraint pas les migrants à abandonner leurs langues et conduit à un "plurilinguisme additionnel» (Vicher, $2011: 10)$. Certes, cela peut différer du développement des compétences plurilingues et de la mobilisation des ressources langagières des personnes, mais cela atteste du climat d'ouverture ambiant, quoique relatif.

\section{Un climat d'ouverture... relatif}

4 La réalité du plurilinguisme est telle dans ces dispositifs de formation, et plus précisément chez les personnes migrantes, qu'il est difficile de le nier et plus avisé/ pertinent de rappeler, à juste titre, combien il est intéressant de s'appuyer sur les langues d'origine et de veiller à ne pas les effacer. Toutefois, certaines préconisations nous invitent à constater la complexité à déroger à la règle du monolinguisme, dont parlait Dabène (1996). Dans les dispositifs auxquels nous faisons référence ${ }^{2}$, et dans lesquels s'ancrent nos réflexions et expériences de recherche, la prise en compte des apprenants dans les formations et la traduction en termes de contenus didactiques génère des doutes, des indifférences voire même le rejet de la part de certains formateurs que nous avons côtoyés au cours de nos recherches.

5 Si, comme l'écrit Puren (2011), une prise en compte des langues d'origine constitue pour le moment davantage un poids supplémentaire qu'un atout « dans la mesure où, faute de bases expérimentales éprouvées par la recherche-action, les acteurs seraient livrés à eux-mêmes " ${ }^{3}$, à l'inverse, la formation linguistique «tout en français » semble s'être imposée sans difficulté, comme une évidence.

6 Restée longtemps officieuse, cette tendance à la formation monolingue se diffuse et s'officialise dans les documents cadre des formations linguistiques rattachés au Contrat d'Intégration Républicaine (CIR) : il est demandé depuis 2015 que les formateurs s'expriment «de manière simple et adaptée à un public allophone, exclusivement en langue française qu'ils doivent parfaitement maitriser »(OFII, 2015 :13 ; OFII, 2019 : 22).

7 Précisons d'abord quelques raisons «fortes» de privilégier un enseignement monolingue, et leurs liens à un arrière-plan idéologique qui s'y prête.

8 Si ces dispositifs officiels s'attachent de façon « évidente » avec cette posture, c'est en partie lié aux enjeux de la formation elle-même, qui est présentée comme devant constituer un point de départ de l'insertion de ces personnes : « Le CIR est la première étape de votre parcours d'intégration en France. Vous devez en respecter les droits et obligations. Il est la garantie d'une intégration réussie dans votre pays d'accueil. » (Contrat d'Intégration Républicaine, $2016: 2$ )

9 Dans le cadre du CIR, différentes modalités de formation visent conjointement à favoriser une exposition maximale à la langue française, c'est-à-dire par une formation :

- précoce et directe (obligatoire),

- intensive de 12 à 30 heures de formation par semaine (OFII, 2019: 15),

- devant s'appuyer « exclusivement sur des documents authentiques » (OFII, 2019: 8-9), 
- et dans cette même logique d'«exposition maximale», une formation strictement monolingue (voir citation supra).

Dans cette optique, l'exposition à la langue française serait favorable à son apprentissage, représentant avant tout les moyens de faire parler les migrants, pour reprendre l'expression d'Anderson (2002). Ces premiers éléments d'interprétation nous permettent de souligner le caractère cohérent (et pour le moins orienté) de cette obligation pédagogique officielle dans le cadre de l'insertion de migrants de façon plus générale. Mais comment se traduit cette préférence monolingue en classe?

\section{Le monolinguisme dans ces formations : orientation, injonction ou panacée?}

\section{Quelques réceptions de l'approche monolingue par ses acteurs}

11 Soulignons que la préférence pour le monolinguisme ne relève pas seulement de la décision des institutions et enseignantee.s. Parmi les différentes classes de formation linguistiques observées au cours de nos recherches (obligatoires ou bien volontaires), pour certains stagiaires voire certains groupes entiers, une formation monolingue représente l'occasion de pratiquer exclusivement le français, et certains stagiaires n'apprécient pas la présence d'autres langues ou que le recours soit valorisé par l' enseignante.

la diversité d'acteurs préconisant le monolinguisme, les observations et entretiens menés nous conduisent à identifier des positionnements très variés qui vont d'un mode tempéré à une interdiction. Plus précisément, le monolinguisme peut relever d'une simple préférence, qui n'exclut pas la traduction tant du côté des stagiaires que de celui de l'enseignante qui admet y recourir lorsque c'est nécessaire. À l'inverse de façon forte, l'interdiction de traduire pour les stagiaires (à l'oral ou à l'écrit) est parfois explicite, l'enseignante les incitant à faire des efforts pour ne pas passer par d'autres langues, et comprendre directement avant de produire en français. Un positionnement monolingue diverge parfois fortement entre ce qui est revendiqué et ce qui est fait en classe : de l'enseignante qui se met à l'anglais en situation d'urgence, au stagiaire qui traduit en permanence sur son cahier. Ainsi, la revendication d'un monolinguisme n'empêche pas des pratiques plurilingues et plurielles, plus ou moins reniées, plus ou moins masquées. Si ces pratiques plurilingues sont parfois tolérées en classe, elles restent difficilement reconnues, acceptées, encouragées, et encore moins, priorisées.

Du cloisonnement des langues à l'absence de sens

13 Les stagiaires dont il va être question $\mathrm{ici}^{4}$ ont pour la plupart plusieurs formateurs, dont au moins un est pro-monolingue. Tous jouent le jeu de la formation et ne laissaient transparaitre aucune réticence forte envers les exigences de ces formations obligatoires. C'est au cours d'entretiens compréhensifs portant sur d'autres questions ou thématiques ${ }^{5}$ que nous avons pu nous saisir de leurs réceptions vis-à-vis de ce qui se dit, se passe et se pratique en classe autour des langues en présence et de l'apprentissage du français.

14 L est une femme ukrainienne qui a vécu à la frontière polonaise. Elle a appris le polonais et le russe lors de formations au cours desquelles les formateurs faisaient par moment de la traduction, de la comparaison de langues. 
$\mathrm{L}$ : Je penche c'est... uh personne, c'est euh quand y'a beaucoup les langues, c'est... beaucoup mieux euh pour le... cours le... cours obligatoires, ou cours privat, c'est euh, à mon avis c'est beaucoup mieux. C'est beaucoup mieux euh communication.

E : Alors du coup, pour certaines personnes, qu'y ait pas du tout leur langue, du coup c'est pas difficile si elles entendent pas du tout?

$\mathrm{L}:$ (...) Beaucoup personnes assises, et euh pas compris qu'est-ce que pour lee professeur, et pas intéressante, ce cours. Pas intéressante. C'est il faut, et c'est tout. Pas intéressante euh parc'que pas comprendre.

(L, entretien $10,1^{\mathrm{er}}$ septembre $\left.2016: 16^{\prime} 33-17^{\prime} 15\right)^{6}$

L, se positionne ici en opposition à cette exclusivité du français, alors comme pro plurilingue. Ses expériences passées s'en retrouvent idéalisées, opposées à ce qu'elle semble recevoir comme une intransigeance monolingue. L'approche monolingue est alors perçue comme contreproductive, la formation devenant un espace d'incompréhension et d'incommunication, marquant l'absence d'autres langues qui pourraient au contraire être mobilisées, mises à contribution.

Pour ce couple d'irakiens réfugiés ( $\mathrm{P}$ et $\mathrm{Q}$ ), le ressenti est similaire : ils disent ne pas tirer parti de la formation exclusivement monolingue.

Tr : Em'dit certes, y'a des gens qui veulent apprendre, ils sont tout le temps attentifs euh, au cours etcetera, mais y'en a d'autres euh, 'fin y s'en fichent un p'tit peu, du coup au bout d'un moment y s'ennuient en, en cours et ils décrochent. Et lui y'm'dit qu'y d'vrait y'avoir une langue partagée, entre, l'enseignant et euh, et les apprenants parc'que, des fois euh, par exemple pour son cas em'dit qu'elle a envie d'poser une question mais elle ose pas pasque toute façon euh quand la, euh, la prof, euh, va répondre, eva e sait qu'e va pas comprendre. Et puis en fait e, e pose pas la question pasque, pasque elle va parler en français elle va rien (X) (C par E)

( $P$ et $Q$, entretien 13, le 9 septembre 2016: 16'24)

Tr : Ils sont pas d'accord. $(P)$ explique que s'il ne comprend pas une phrase, on peut lui répéter 100 fois il ne la retiendra jamais. Par contre si il comprend euh, le sens de la phrase, tu vas m'la répéter 3 fois j'vais m'en souvenir. Et euh, pour elle euh, em'dit c'est pareil en fait. (...) De cette façonlà, elle retient les mots en fait. Tout en français, ils « apprennent moins ». Les autres langues sont « comme un support ».

(Ibid. : 27 '00 - 30'00 )

(...) Celui qui comprend il va traduire au reste de la promo.

( $P$ et $Q$, entretien 13, le 9 septembre $2016: 32$ '55)

$\mathrm{Au}$ regard de leur connaissance de l'anglais et de leur volonté de s'améliorer en français, tous deux soulignent la nécessité de recourir à des stratégies plurilingues pour appréhender la nouvelle langue à apprendre. À leurs yeux, la traduction donne du sens, participe à la valorisation de soi et de ses compétences. Pour eux aussi, l'exclusivité du français tend à être synonyme de «frein » à la compréhension, à la communication, et à l'apprentissage en classe. Cette réception nous semble assez ironique et nous interpelle tout autant que les témoignages suivants, d'apprenants qui établissent des liens entre l'injonction au français en formation linguistique OFII, et le monolinguisme de l'école :

$\mathrm{E}:$ Mais vous avez peur qu'il oublie l'arabe?

I : Yes yes yes... This is my mother... language ! (Rire) (5'56)

Alors par compensation, à la maison J parle exclusivement arabe avec sa fille : 
Tr : En fait y'm dit (En parlant de J) euh... Y'm dit que euh sa fille quand elle va

à l'école, elle lui parle en français, et lui il lui répond en arabe, (C par E)

$\mathrm{E}: \mathrm{mh}$ mh pour que ça se perde pas.

$\mathrm{Tr}$ : pour que ça se perde pas oui. (11'56)

À travers l'exemple de ces deux réfugiés soudanais (I et J), on parvient à comprendre, non sans surprise, que le père de famille $(\mathrm{J})$ réinvestit à la maison la concurrence qui lui est renvoyée entre les langues, représentées comme cloisonnées. Il se créé alors une opposition, une concurrence linguistique qui vient se rejouer en dehors de la classe. Le tout en français semble venir signifier un choix qu'il s'agirait de faire entre les langues et les cultures.

Si comme évoqué le monolinguisme fait écho de façon forte à la vision de l'intégration dans laquelle il s'inscrit, est-ce que la crainte de ces stagiaires ne serait pas précisément que ce monolinguisme ne soit le signe d'une assimilation linguistique prochaine ? Ces informateurs interrogent également la limite de l'enseignement/apprentissage du français, faisant état d'un rejet relativement explicite du dispositif de formation de façon plus générale :

$\mathrm{J}$ en arabe

Tr.: Il dit que pour lui que l'OFII il mise plus sur la quantité que sur la qualité. Et il dit qu'ils peuvent même lui mettre 200h par jour, si il n'y a pas la qualité, ben il voit pas l'intérêt quoi. (...) (53’56)

À travers une même logique d'exposition maximale à la langue 㡠 comme "moyen de faire parler " la langue cible 㡠, la dimension affective des langues en présence est évacuée, au profit de leur (seule) dimension communicative. Si cette destitution de la dimension affective des langues a déjà fait l'objet de réflexions en didactique des langues étrangères ${ }^{7}$, la tendance à un monolinguisme strict semble y participer de façon logique en faisant taire les autres langues, invisibilisant par conséquent un ensemble de représentations qui y sont attachées. Faire taire pour tendre à mieux faire parler, voilà tout le paradoxe d'un monolinguisme strict en formation linguistique en faveur de l'insertion.

Ces extraits soulignent que derrière l'évidence monolingue se trouve en creux l'idée

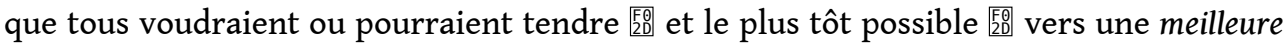
maitrise, ils pourraient directement viser à se transformer en français, sans transition $n i$ mélange, indépendamment des représentations des langues en jeu dans ces apprentissages. Finalement, les entretiens ont permis de co-construire des représentations d'une importance potentiellement cruciale pour l'apprentissage $\mathrm{du}$ français de ces stagiaires, représentations restées invisibles, et que ce cadre d'intégration linguistique n'avait pas permis de discuter ou mettre à profit en formation.

\section{Le plurilinguisme : des pratiques « en plus » qui interrogent}

Pour d'autres dispositifs existants, il est fréquent qu'une place soit faite aux pratiques plurilingues et à la diversité linguistique et culturelle. Même si le français est privilégié, d'autres langues résonnent dans ces formations moins contraintes par le temps et par les directives des contrats d'intégration. Toutefois, il s'agit d'une place réservée à des activités parallèles, dites « en plus ", qui viennent compléter les cours de français tels 
des ateliers d'écriture ou des ateliers de lecture. La mise en place de ces espace-temps, où les langues se délient, s'entend comme l'opportunité d'admettre et de faire vivre une pluralité linguistique qui permet également de sortir du français fonctionnel, des dimensions pragmatiques et communicatives ainsi que de la forme scolaire. Il s'agit, le plus souvent, de valoriser les langues en présence et les expériences migratoires et d'en partir pour construire, collectivement ou individuellement, des écrits nourris de ces histoires en langues. Il est alors aisé d'observer une prise de conscience de la diversité des répertoires linguistiques, tant pour les apprenants que pour les formateurs, et, dans une certaine mesure, une mobilisation de ces ressources. L'alternance, la traduction, les discussions autour des rapports aux langues des participants aux ateliers, sont autant d'éléments qui témoignent de l'ouverture au plurilinguisme. Il en va de même avec l'activité de lecture qui sollicite davantage les apprenants à partager leurs langues. À travers les pratiques observées dans ces ateliers, il apparait que la dimension identitaire des langues soit mise en évidence. D'une certaine manière, on pourrait penser que ce type d'activités pallie ou compense les conséquences des formations tout en français. Pour autant, des incompréhensions et des résistances ont également lieu, notamment parce que ces activités peuvent parfois être intrusives ou déconnectées des objectifs ou besoins des personnes qui viennent apprendre le français. Les plurilinguismes semblent demeurer difficiles à reconnaitre, quel que soit le contexte de formation, dans la mesure où il faut que cela fasse sens pour les personnes et qu'elles ne ressentent pas d'injonction ou de prescription au plurilinguisme.

\section{Ouverture des acteurs au plurilinguisme dans une perspective appropriative}

20 À nos yeux, l'accompagnement de ces apprenants ne relève ni d'une unique bonne pratique ni d'une bonne méthode, qu'elle soit (pro)monolingue ou (pro)plurilingue. Manifestement, il semble que ce soit avant tout le dispositif ou la démarche du formateur qui est au centre, et non les personnes, en l'occurrence les bénéficiaires de ces formations : les logiques de ce dispositif pouvant parfois se heurter à leurs propres rapports aux langues, stratégies et expériences. Ainsi, raisonner en termes de bonnes et mauvaises pratiques mono- ou plurilingues conduirait à :

- prendre le risque de délégitimer et déconsidérer des stratégies d'apprentissages plurilingues pourtant expérimentées par certains apprenants (en classe ou non, dans l'appropriation de langues déjà-là, et dont le français fait parfois déjà partie) ;

- déposséder certains migrants du sentiment d'être l'auteur principal des processus d'appropriation qu'ils vivent ou bien appréhendent.

21 L'exposition maximale au français vient alors aisément symboliser une centration sur la formation où c'est l'apprentissage guidé en français avant tout qui devrait, pourrait, venir constituer la solution à l'apprentissage, voire à la maîtrise de la langue. Cela peut être sensiblement la même chose avec des approches plurilingues qui ne font pas sens pour les acteurs de la formation linguistique (formateurs et apprenants) et tendent à être perçues comme une nouvelle injonction, laquelle se voudrait tout autant bénéfique, pertinente et bienveillante pour les apprenants. En effet, il semble vain pour des formateurs de ce type de formation linguistique à visée d'insertion, de se contenter 
d'intégrer à leurs enseignements des outils plurilingues qui risqueraient de représenter

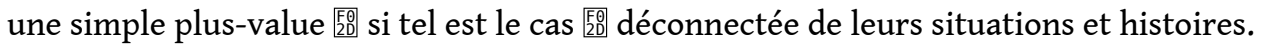

En somme, il conviendrait de permettre à tous les acteurs de ces formations 医 formateurs comme apprenants 㡠 d'avoir prise lorsqu'ils ne sont pas en accord avec les approches promues 頤 voire préconisées 医 et de se sentir être les auteurs véritables de leurs enseignements et apprentissages. Quelle approche du plurilinguisme permettrait d'allier la reconnaissance de langues en jeu pour ces processus à celle des projets individuels d'appropriation et/ou d'intégration?

Les approches biographiques et les approches plurielles ont pu faire leur preuve dans le sens où des travaux, comme ceux réalisés par Bretegnier $(2009,2013)$, ont montré que l'introduction d'activités plurilingues offrait aux apprenants l'opportunité de mobiliser leurs expériences linguistiques et culturelles et de " déplacer l'accent de l'apprentissage d'une "langue-cible", à la construction plus large de ressources plurielles» (Ibid., 2013: 308). Toutefois, ces réponses pertinentes, pour qui souhaite s'appuyer sur le répertoire plurilingue, ne font pas toujours sens et c'est en cela qu'il convient de continuer à s'interroger sur les usages des outils et approches dont des institutions et acteurs engagés dans des formations linguistiques, ne parviennent pas à s'emparer. Au regard de ces entretiens, observations et expériences, nous pensons qu'il serait pertinent d'opter pour une alternative, qui consisterait à sortir d'une didactique du plurilinguisme pensée essentiellement en termes d'outils et d'approches plurielles. Des outils qui certes, peuvent se révéler stimulants, mais qui, selon nous, tendent à mettre l'accent sur des techniques à greffer à un cadre, sans prendre le temps de les mettre en question. En outre, ils ne permettent pas, si tant est que cela soit possible, d'éviter l'écueil des bonnes pratiques ou encore d'éviter de venir représenter une contrainte supplémentaire.

Il s'agirait alors de faire un pas de côté, en empruntant un chemin autre, moins confortable, plus instable, où l'appropriation n'est plus un simple synonyme plus élégant de la maitrise de la langue (Klinkenberg, 2015) ni un objectif à atteindre, un résultat, mais la possibilité de laisser advenir la langue à soi (Castellotti, 2017) en s'engageant à accompagner sans avoir déterminé par avance le chemin à suivre, en laissant la possibilité de (se) transformer. L'idée n'est pas tant de renoncer à des démarches favorables à la diversité linguistique ou au décloisonnement des langues, que de renoncer à mettre en application des techniques qui ne font pas sens ou qui ne correspondent pas aux attentes des personnes. Penser essentiellement la didactique 醫 des langues et du plurilinguisme 臨 à partir de techniques, d'outils clés en main et méthodes tend à mettre essentiellement l'accent sur la dimension pragmatique (Debono, 2019), et cela au détriment de la dimension ontologique des pratiques, des usages et des représentations des langues. Autrement dit, se laisser rattraper par les recettes et la recherche de bonnes pratiques (pro)mono- ou (pro)pluri-lingues écarte la possibilité de concevoir les langues comme «expérience du monde (perceptioninterprétation-relation) » (Castellotti, $2017: 90)$.

S'autoriser à se laisser transformer par ce qui se passe en formation (imprévus, réactions, productions, retours, etc.) nécessite assez inévitablement pour le formateur de se laisser soi-même troubler, déstabiliser, transformer par les rencontres faites. Le monolinguisme strict vient selon nous aisément signifier à l'inverse, que « le pas à faire vers l'autre » est indiscutablement d'abord celui du migrant : ce serait à lui et d'abord à 
lui de s'adapter, de faire l'effort de comprendre dans le cadre d'une méthodologie considérée comme plus efficace a priori.

\section{Pour ne pas conclure...} de ces possibilités, se sentent légitimes à faire entrer leurs propres expériences plurilingues dans leur pratique. En outre, il serait nécessaire que ces formateurs aient une plus grande souplesse et la souplesse liberté (mais aussi par extension, l'éthique, voire, la responsabilité ?) pédagogiques et didactiques nécessaires pour se laisser emmener dans de telles expériences de façon réflexive et assumée, notamment dans des dispositifs d'intégration qui représentent et institutionnalisent une idéologie monolingue qui met hors de portée le plurilinguisme et les réflexions qui s'y rapportent.

\section{BIBLIOGRAPHIE}

Anderson, P. (2002). « Nous avons les moyens de vous faire parler »... ou : ce que devient la pragmatique linguistique en didactique des langues. Semen. Revue de sémio-linguistique des textes et discours [En ligne], 14, mis en ligne le 30 avril 2007, consulté le 15 novembre 2020. URL : http:// semen.revues.org/2537.

Anderson, P., \& Laseldi-Grelis. (2003). De la langue originaire à la langue de l'autre. Ela. Etudes de linguistique appliquée, 131, 3, 343-356.

Beacco, J.-C. (2008). Les langues dans les politiques d'intégration des migrants adultes. Division des Politiques Linguistiques, Strasbourg : Conseil de l'Europe. 
Bretegnier, A. (2009). Histoires de langues en formation. Une démarche de rechercheintervention alter-réflexive. In M. Molinié \& E. Huver (coord.), Praticiens-chercheurs à l'écoute du sujet plurilingue. Réflexivité et interaction biographique en sociolinguistique et en didactique. Carnets d'Atelier de Sociolinguistique, 4. (pp. 35-63). LESCLaP, Université Picardie Jules Verne.

Bretegnier, A. (2013). Formation linguistique des adultes : Vers une didactique des langues en relations. In V. Bigot, A. Bretegnier, \& M.-T. Vasseur (dirs.), Vers le plurilinguisme ? 20 ans après (pp. 301-310). Paris : Ed. des Archives Contemporaines.

Castellotti, V. (2017). Pour une didactique de l'appropriation : diversité, compréhension, relation. Paris : Didier.

Coste, D. (2010). Diversité des plurilinguismes et formes de l'éducation plurilingue et interculturelle. Cahiers de l'ACEDLE, vol7, 1,141-165.

Dabène, L. (1996). Des migrants et des langues. Ecarts d'identité, 76, 2-3.

Debono, M. (2019). L'utilitarisme et sa critique en didactique des langues : les frontières du rationnel. Actes de colloque Dipralang, 1 / 2019, TDLFE. https://revue-tdfle.fr/actes-1-44/162-1utilitarisme-et-sa-critique-en-didactique-des-langues-les-frontieres-du-rationnel consulté le 30 mai 2020.

Hambye, P., \& Romainville, A. (2014). Apprentissage du français et intégration. Des évidences à interroger. Bruxelles : EME et Intercommunications.

Kauffman, J.-C. (1996). L'entretien compréhensif. Paris : Nathan.

Klinkenberg, J.-M. (2015). La langue dans la cité. Vivre et penser l'équité culturelle. Bruxelles : Les impressions nouvelles.

Lebreton, E. (2017). Pour des formations linguistiques (trans)formatrices : renverser les évidences pour penser l'appropriation du français par des adultes migrants. Thèse de doctorat soutenue le 11 septembre 2017 à l'Université de Rouen.

Lüdi, G. (2002). Pour une linguistique de la compétence du locuteur plurilingue. Revue française de linguistique appliquée, vol.9, 125-135.

Mercier, E. (2020). Formations linguistiques contractuelles et intégration d'adultes migrants : Quelle pertinence à l'obligation de formation? Thèse de doctorat, Université de Tours, soutenue le 16 décembre 2020 à l'Université de Tours.

OFII. (2015). Marché $n^{\circ} 1524007$, Cahier des clauses particulières - Formation linguistique.

OFII. (2019). Marché $n^{\circ} 190001$, Cahier des clauses particulières - Formation linguistique.

Puren, C. (2011). Français Langue d'Intégration - droit de réponse. https://www.christianpuren.com/ fli/ consulté le 12 mai 2020

Vicher, A. et al. (2011). Référentiel Langue d'Intégration. Direction de l'Accueil, de l'Intégration et de la Citoyenneté.

\section{NOTES}

1. https://rm.coe.int/doc-16-portrait-plurilingue-une-tache-reflexive-pour-les-volontaires-a/ 168075aaaf consulté le 23 mai 2020.

2. Il s'agit d'une part, de formations prescrites, obligatoires selon leur rattachement à un projet professionnel (Pôle emploi et la Région comme prescripteurs) ou à un contrat d'intégration (CAI 
ou CIR, selon les dates de nos recherches ; formations assurées par l'OFII, opérateur de l'État en matière d'immigration); et d'autre part, d'actions linguistiques menées dans des associations de quartier, reposant exclusivement sur le volontariat. Ces types de dispositifs partagent l'objectif de favoriser l'insertion sociale et professionnelle des personnes à travers l'apprentissage, voire la maîtrise de la langue française.

3. https://www.christianpuren.com/fli/ consulté le 12 mai 2020.

4. Ces entretiens ont été réalisés dans le cadre d'une recherche doctorale (voir Mercier, 2020) qui se déroule dans un organisme de formation dans lequel le chercheur est également formateur de ces formations linguistiques obligatoires. 14 stagiaires y ont été interrogés.

5. En adoptant une méthodologie compréhensive inspirée des travaux de J.-C. Kauffman (1996), il s'agissait avant tout de s'interroger sur leur présence et leur intégration en France ainsi que sur le rôle de la formation en langue dans leur apprentissage du français, avec parfois l'aide d'interprètes professionnels ( $\mathrm{Tr}$ dans les extraits qui suivent).

6. $\mathrm{E}$ : enquêteur (Eric Mercier); Tr: interprète; (C) : coupé par un interlocuteur ; $(\mathrm{X})$ : mot incompréhensible ou inintelligible. La transcription réalisée vise à privilégier ce qui est entendu.

7. Voir par exemple Anderson \& Laseldi-Grelis, 2003.

\section{RÉSUMÉS}

Les formations linguistiques pour adultes migrants sont marquées par diverses préconisations qui témoignent d'une relative ouverture au plurilinguisme. Pour les formateurs et les apprenants rencontrés dans nos recherches, le plurilinguisme et la didactique qui peut en résulter s'appréhendent de diverses manières selon les expériences, les projets et les conceptions des langues et l'enseignement-apprentissage du français dans ce contexte de formation-insertion. $\mathrm{Au}$ gré des réflexions, il apparait que la didactique du plurilinguisme ne peut se limiter à être pensée en termes d'outillage des acteurs des formations (formateurs et apprenants), ni en termes de bonnes ou mauvaises pratiques qui conduiraient à prescrire/proscrire les plurilinguismes. En s'inscrivant dans une perspective appropriative, penser la didactique du plurilinguisme revient à prendre appui sur les expériences, sur leurs sens et sur les transformations qu'elles génèrent.

Language training for adult migrants is characterized by various recommendations which show a relative openness to plurilingualism. For the teachers and learners we met in our research, plurilingualism and the didactics that can result from it can be viewed in different ways depending on experiences, projects and conceptions of languages as well as the teachinglearning of French in this context of training-insertion. In the course of the discussions, it appears that the teaching of plurilingualism cannot be considered in terms of educative tools used by teachers and learners, neither in terms of good or bad practices that would lead to prescribe/proscribe plurilingualism. From an appropriative approach, to reflect didactics of plurilingualism means to rely on experiences, on their meanings and on the transformations they generate. 


\section{INDEX}

Mots-clés : Formations linguistiques, adultes migrants, plurilinguismes, expériences, appropriation

Keywords : Language training, adult migrants, plurilingualism, experiences, appropriation

\section{AUTEURS}

\section{EMILIE LEBRETON}

Aix-Marseille Université, CNRS, Laboratoire Parole et Langage (LPL)

Emilie Lebreton est maîtresse de conférences en Didactique du FLE, ses recherches portent sur l'appropriation du français en contexte migratoire. Les recherches collaboratives menées avec les acteurs diversement impliqués auprès de personnes migrantes engagées en formation linguistique, la conduisent à développer des réflexions concernant l'articulation langue(s) et insertion(s), la dimension langagière de la scolarisation et de la socialisation, les conceptions du métier d'enseignant/formateur de FLE/S.

emilie.lebreton[at]univ-amu.fr

\section{ÉRIC MERCIER}

Université de Tours, EA4428 DYNADIV

Éric Mercier est docteur en Sociolinguistique et Didactique du FLE/S. Ses recherches interrogent la pertinence des politiques de formation linguistique pour adultes migrants en s'intéressant aux réceptions de différents acteurs impliqués, et avec comme angles principaux l'articulation langue(s)/insertion(s), les imaginaires linguistiques et nationaux liés à l'insertion et à l'obligation formative.

eric.mercier[at]univ-tours.fr 